\title{
Pentagonal Cone b-metric Spaces over Banach Algebras and Fixed Point Theorem of Generalized Lipschitz Mapping
}

\author{
Abba Auwalu ${ }^{1, *}$ \\ ${ }^{1}$ Department of Mathematics, Near East University, Nicosia, via Mersin 10, Turkey
}

\begin{abstract}
In this paper, we introduce the concept of pentagonal cone $b$-metric space over Banach algebras as a generalization of cone metric space over Banach algebras and many of its generalizations. Furthermore, we prove Banach fixed point theorem in such a space. Our result unify, complement and/or generalized some recent results in the papers [1-7], and many others. We provide some examples to elucidate the validity and superiority of our results.
\end{abstract}

\section{Introduction}

In 2013, Huang and Shao [1] instigated the idea of cone metric space with a Banach algebra by replacing the complete normed space in cone metric space with a Banach algebra. They established that cone metric spaces over Banach algebras are not equal to metric spaces in terms of existence of the fixed points of mappings. Further, they proved some fixed point results for various contractive conditions in such a space. Subsequently, many authors established interesting and significant results in a cone metric space over Banach algebras and its generalizations (see; [2], [3], [4], [5], [7] and references therein).

\section{Preliminaries}

In this part, we provide some definitions and results required in the consequence.

Definition 2.1 ([1]) A subset $\mathcal{P}$ of Banach algebra $\mathcal{B}$ is called a cone if:

(1) $\mathcal{P}$ is nonempty closed and $\{\theta, e\} \subset \mathcal{P}$; (2) $k_{1} \mathcal{P}+k_{2} \mathcal{P} \subset \mathcal{P}$ for all nonnegative real numbers $k_{1}, k_{2}$; (3) $\mathcal{P}^{2}=\mathcal{P P} \subset \mathcal{P}$; (4) $\mathcal{P} \cap(-\mathcal{P})=\{\theta\}$, where $\theta$ and $e$ denote the zero and unit elements of the Banach algebra $\mathcal{B}$, respectively. For a given cone $\mathcal{P} \subset \mathcal{B}$, we write $y \leqslant z$ if and only if $z-y \in \mathcal{P}$, where $\leqslant$ is a partial order relation defined on $\mathcal{P}$. We shall write $y<z$ to indicate that $y \leqslant z$ but $y \neq z$, while $y \ll z$ will stand for $z-y \in$ int $\mathcal{P}$, where int $\mathcal{P}$ denotes the interior of $\mathcal{P}$. If int $\mathcal{P} \neq \varnothing$ then $\mathcal{P}$ is called a solid cone.

Definition 2.2 ([2]) Let $\mathcal{P}$ be a solid cone in a Banach algebra $\mathcal{B}$. A sequence $\left\{z_{i}\right\} \subset \mathcal{P}$ is said to be a c-sequence if for each $\theta \ll c$ there exists $n_{0} \in \mathbb{N}$ such that $z_{i} \ll c$ for all $i>n_{0}$.

Lemma 2.3 ([2]) Let $\mathcal{P}$ be a solid cone in a Banach algebra $\mathcal{B}$ and $\left\{z_{i}\right\} \subset \mathcal{P}$ be a sequence with $\left\|z_{i}\right\| \rightarrow 0(i \rightarrow \infty)$, then for each $\theta \ll c$, there exists $n_{0} \in \mathbb{N}$ such that for all $i>n_{0}$, we have $z_{i} \ll c$.

\footnotetext{
*e-mail: abba.auwalu@ neu.edu.tr,abbaauwalu@yahoo.com
} 
Lemma 2.4 ([8]) Let $\mathcal{B}$ be a Banach algebra with a unit e and $\tau \in \mathcal{B}$. If the spectral radius $\delta(\tau)$ of $\tau$ is less than one, i.e. $\delta(\tau)=\lim _{n \rightarrow \infty}\left\|\tau^{n}\right\|^{\frac{1}{n}}=i n f_{n \in \mathbb{N}}\left\|\tau^{n}\right\|^{\frac{1}{n}}<1$, then $(e-\tau)$ is invertible in $\mathcal{B}$. Moreover, $(e-\tau)^{-1}=\sum_{j=0}^{\infty} \tau^{j}$.

Remark 2.5 ([2]) If the spectral radius $\delta(\tau)<1$, then $\left\|\tau^{i}\right\| \rightarrow 0(i \rightarrow \infty)$.

Lemma 2.6 ([4]) Let $\mathcal{B}$ be a real Banach algebra with a solid cone $\mathcal{P}$. For $a, b, c, \tau \in \mathcal{P}$, if (1) $a \leqslant b \ll c$, then $a \ll c$. (2) $a \leqslant \tau a$ and $\delta(\tau)<1$, then $a=\theta$.

\section{Main Results}

In this part, we firstly instigate the idea of a pentagonal cone $b$-metric space over a Banach algebra and provide an example showing that this concept is very tremendous than pentagonal cone metric space over a Banach algebra [7]. Secondly, we describe convergence and a Cauchy sequence in a pentagonal cone $b$-metric space over a Banach algebra and then prove Banach fixed point theorem in this space.

Definition 3.1 Let $\mathcal{Z}$ be a nonempty set and $\mathcal{B}$ be a Banach algebra. Suppose that $\rho$ : $\mathcal{Z} \times \mathcal{Z} \rightarrow \mathcal{B}$ is a mapping satisfying, for all $x, y, z, u, v \in \mathcal{Z}, x \neq z, z \neq u, u \neq v, v \neq y$, the following conditions:

$\left(P b_{1}\right) \quad \theta<\rho(x, z)$ and $\rho(x, z)=\theta \Leftrightarrow x=z$;

$\left(P b_{2}\right) \rho(x, z)=\rho(z, x)$;

$\left(P b_{3}\right)$ there exist $k \in \mathcal{P}, e \leqslant k$ such that $\rho(x, y) \leqslant k[\rho(x, z)+\rho(z, u)+\rho(u, v)+\rho(v, y)]$.

Then $\rho$ is called a pentagonal cone $b$-metric on $\mathcal{Z}$, and $(\mathcal{Z}, \rho)$ is called a pentagonal cone $b$-metric space over Banach algebra $\mathcal{B}$.

Remark 3.2 A pentagonal cone b-metric space over a Banach algebra $\mathcal{B}$ is also pentagonal cone metric space over Banach algebra $\mathcal{B}$ where $k=1$. For $k>1$, the converse is not necessarily true, see the following example.

Example 3.3 Let $\mathcal{B}=\left\{a=\left(a_{m n}\right)_{2 \times 2}: a_{m n} \in \mathbb{R}, 1 \leq m, n \leq 2\right\}$, $\|a\|=\sum_{1 \leq m, n \leq 2}\left|a_{m n}\right|$. Then $\mathcal{B}$ is a real Banach algebra with a unit element $e=\left(\begin{array}{ll}1 & 0 \\ 0 & 1\end{array}\right)$ and zero element $\theta=\left(\begin{array}{ll}0 & 0 \\ 0 & 0\end{array}\right)$.

The set $\mathcal{P}=\left\{a \in \mathcal{B}: a_{m n} \geq 0,1 \leq m, n \leq 2\right\}$ is a solid cone in $\mathcal{B}$. Let $\mathcal{Z}=\{1,2,3,4,5\}$, and mapping $\rho: \mathcal{Z} \times \mathcal{Z} \rightarrow \mathcal{B}$ is define as:

$$
\begin{gathered}
\rho(z, z)=\left(\begin{array}{ll}
0 & 0 \\
0 & 0
\end{array}\right), \text { for all } z \in \mathcal{Z} ; \\
\rho(1,2)=\rho(2,1)=\left(\begin{array}{cc}
6 & 6 \\
10 & 10
\end{array}\right) ; \\
\rho(1,3)=\rho(3,1)=\rho(1,4)=\rho(4,1)=\rho(2,3)=(3,2)=\rho(2,4)=\rho(4,2)=\rho(3,4)=\rho(4,3)=\left(\begin{array}{cc}
\frac{1}{2} & \frac{1}{2} \\
1 & 1
\end{array}\right) ; \\
\rho(1,5)=\rho(5,1)=\rho(2,5)=\rho(5,2)=\rho(3,5)=\rho(5,3)=\rho(4,5)=\rho(5,4)=\left(\begin{array}{ll}
2 & 2 \\
3 & 3
\end{array}\right) .
\end{gathered}
$$

Then $(\mathcal{Z}, \rho)$ is a pentagonal cone b-metric space over Banach algebra $\mathcal{B}$ with coefficient $k=\left(\begin{array}{ll}2 & 0 \\ 0 & 2\end{array}\right)$. But $(\mathcal{Z}, \rho)$ is not a pentagonal cone metric space over Banach algebra $\mathcal{B}$ 
because it lacks the pentagonal property $\left(P_{3}\right)$ in [7] as follows:

$$
\begin{aligned}
\left(\begin{array}{cc}
6 & 6 \\
10 & 10
\end{array}\right) & =\rho(1,2)>\rho(1,3)+\rho(3,4)+\rho(4,5)+\rho(5,2) \\
& =\left(\begin{array}{ll}
\frac{1}{2} & \frac{1}{2} \\
1 & 1
\end{array}\right)+\left(\begin{array}{ll}
\frac{1}{2} & \frac{1}{2} \\
1 & 1
\end{array}\right)+\left(\begin{array}{ll}
2 & 2 \\
3 & 3
\end{array}\right)+\left(\begin{array}{ll}
2 & 2 \\
3 & 3
\end{array}\right)=\left(\begin{array}{ll}
5 & 5 \\
8 & 8
\end{array}\right), \\
& \text { as }\left(\begin{array}{cc}
6 & 6 \\
10 & 10
\end{array}\right)-\left(\begin{array}{ll}
5 & 5 \\
8 & 8
\end{array}\right)=\left(\begin{array}{ll}
1 & 1 \\
2 & 2
\end{array}\right) \in \mathcal{P} .
\end{aligned}
$$

Definition 3.4 Let $(\mathcal{Z}, \rho)$ be a pentagonal cone b-metric space with Banach algebra $\mathcal{B}, z \in \mathcal{Z}$ and $\left\{z_{i}\right\}$ be a sequence in $(\mathcal{Z}, \rho)$. Then we say

(1) $\left\{z_{i}\right\}$ converges to $z$ if, for each $c \in \mathcal{B}$ with $\theta \ll c$, there is a natural number $n_{0}$ such that $\rho\left(z_{i}, z\right) \ll c$ for all $i \geq n_{0}$. We denote this by $z_{i} \rightarrow z(i \rightarrow \infty)$.

(2) $\left\{z_{i}\right\}$ is a Cauchy sequence if, for each $c \in \mathcal{B}$ with $\theta \ll c$, there is a natural number $n_{0}$ which is independent of $n$ such that $\rho\left(z_{i}, z_{i+n}\right) \ll c$ for all $i \geq n_{0}$.

(3) $(\mathcal{Z}, \rho)$ is said to be complete if every Cauchy sequence in $(\mathcal{Z}, \rho)$ is convergent.

Theorem 3.5 Let $(\mathcal{Z}, \rho)$ be a complete pentagonal cone b-metric space over Banach algebra $\mathcal{B}$ with $\theta \leqslant k$ and $\mathcal{P}$ be a solid cone in $\mathcal{B}$. Suppose that $F: \mathcal{Z} \rightarrow \mathcal{Z}$ is a mapping satisfying the generalized Lipschitz condition:

$$
\rho(F z, F y) \leqslant \tau \rho(z, y), \text { for all } z, y \in \mathcal{Z},
$$

where $\tau \in \mathcal{P}$ such that the spectral radius $\delta(\tau)<1$. Then $F$ has a unique fixed point $z^{*}$ in $\mathcal{Z}$. Moreover, for any $z_{0} \in \mathcal{Z}$, the iterative sequence $\left\{F^{i} z_{0}\right\}(i \in \mathbb{N})$ converges to $z^{*}$.

Proof. Let $z_{0}$ be arbitrary point in $\mathcal{Z}$. We define a sequence $\left\{z_{i}\right\}$ in $(\mathcal{Z}, \rho)$ by

$$
z_{i+1}=F z_{i}=F^{i+1} z_{0}, \text { for all } i \in \mathbb{N} \text {. }
$$

Suppose $z_{j}=z_{j+1}$ for some $j \in \mathbb{N}$, then $z^{*}=z_{j}=F^{j} z_{0}$ is a fixed point of $F$. Further, for any $z_{0} \in \mathcal{Z}$, the iterative sequence $\left\{F^{j} z_{0}\right\}(j \in \mathbb{N})$ converges to $z^{*}$ and the result is proved. Hence, we assume that $z_{i} \neq z_{i+1}$ for all $i \in \mathbb{N}$. We shall show that $\left\{z_{i}\right\}$ is a Cauchy sequence in $(\mathcal{Z}, \rho)$. To achieve our goal, we split the proof via three cases as follows:

Case 1. Let $\delta(\tau) \in\left[0, \frac{1}{k}\right.$ ) for $k>1$. Now, using (1) and (2), we have

$$
\rho\left(z_{i}, z_{i+1}\right)=\rho\left(F z_{i-1}, F z_{i}\right) \leqslant \tau \rho\left(z_{i-1}, z_{i}\right)<\rho\left(z_{i-1}, z_{i}\right),
$$

that is, the sequence $\left\{\rho\left(z_{i}, z_{i+1}\right)\right\}$ is strictly decreasing, and from this it follows that $\rho\left(z_{i}, z_{i+1}\right) \neq$ $\rho\left(z_{j}, z_{j+1}\right)$ whenever $i \neq j$. Hence, from (1), (2) and (3), we obtain

$$
\begin{aligned}
\rho\left(z_{i}, z_{i+1}\right) & \leqslant \tau \rho\left(z_{i-1}, z_{i}\right)=\tau \rho\left(F z_{i-2}, F z_{i-1}\right) \leqslant \tau^{2} \rho\left(z_{i-2}, z_{i-1}\right)=\tau^{2} \rho\left(F z_{i-3}, F z_{i-2}\right) \\
& \leqslant \tau^{3} \rho\left(z_{i-3}, z_{i-2}\right) \leqslant \cdots \leqslant \tau^{i} \rho\left(z_{0}, z_{1}\right), \text { for all } i \in \mathbb{N} .
\end{aligned}
$$

Similarly, $\quad \rho\left(z_{i}, z_{i+2}\right) \leqslant \tau^{i} \rho\left(z_{0}, z_{2}\right)$, for all $i \in \mathbb{N}$.

Since $\delta(\tau)<\frac{1}{k}$, then $\delta\left(k \tau^{3}\right) \leq k \delta\left(\tau^{3}\right) \leq k \delta(\tau) \delta(\tau) \delta(\tau)<\frac{1}{k^{2}}<1$ and by Lemma 2.4, it follows that $\left(e-k \tau^{3}\right)$ is invertible in $\mathcal{B}$. Moreover,

$$
\left(e-k \tau^{3}\right)^{-1}=\sum_{j=0}^{\infty}\left(k \tau^{3}\right)^{j}, \text { and similarly }\left(\frac{e}{k}-\tau\right)^{-1}=\sum_{j=0}^{\infty} k^{j+1} \tau^{j}
$$


Also since $\delta(\tau)<\frac{1}{k}<1$, by Remark 2.5, we obtain that $\quad\left\|\tau^{i}\right\| \rightarrow 0(i \rightarrow \infty)$.

Now, we consider $\rho\left(z_{i}, z_{i+n}\right)$ in two cases:

Case 1.1. If $n$ is odd, say $n=2 j+1(j \in \mathbb{N})$, then, by using $\left(P b_{3}\right),(4),(6)$ and the fact that $\rho\left(z_{i}, z_{i+1}\right) \neq \rho\left(z_{j}, z_{j+1}\right)$ whenever $i \neq j$, we obtain that

$$
\begin{aligned}
\rho\left(z_{i}, z_{i+2 j+1}\right) \leqslant & k\left[\rho\left(z_{i}, z_{i+1}\right)+\rho\left(z_{i+1}, z_{i+2}\right)+\rho\left(z_{i+2}, z_{i+3}\right)\right]+k \rho\left(z_{i+3}, z_{i+2 j+1}\right) \\
\leqslant & k\left[\rho\left(z_{i}, z_{i+1}\right)+\rho\left(z_{i+1}, z_{i+2}\right)+\rho\left(z_{i+2}, z_{i+3}\right)\right] \\
& +k^{2}\left[\rho\left(z_{i+3}, z_{i+4}\right)+\rho\left(z_{i+4}, z_{i+5}\right)+\rho\left(z_{i+5}, z_{i+6}\right)\right]+k^{2} \rho\left(z_{i+6}, z_{i+2 j+1}\right) \\
\leqslant & k\left[\rho\left(z_{i}, z_{i+1}\right)+\rho\left(z_{i+1}, z_{i+2}\right)+\rho\left(z_{i+2}, z_{i+3}\right)\right] \\
& +k^{2}\left[\rho\left(z_{i+3}, z_{i+4}\right)+\rho\left(z_{i+4}, z_{i+5}\right)+\rho\left(z_{i+5}, z_{i+6}\right)\right] \\
& +k^{3}\left[\rho\left(z_{i+6}, z_{i+7}\right)+\rho\left(z_{i+7}, z_{i+8}\right)+\rho\left(z_{i+8}, z_{i+9}\right)\right]+\cdots \\
& +k^{\frac{2 j}{3}}\left[\rho\left(z_{i+2 j-3}, z_{i+2 j-2}\right)+\rho\left(z_{i+2 j-2}, z_{i+2 j-1}\right)+\rho\left(z_{i+2 j-1}, z_{i+2 j}\right)\right] \\
& +k^{\frac{2 j}{3}} \rho\left(z_{i+2 j}, z_{i+2 j+1}\right) \\
\leqslant & k\left[\tau^{i} \rho\left(z_{0}, z_{1}\right)+\tau^{i+1} \rho\left(z_{0}, z_{1}\right)+\tau^{i+2} \rho\left(z_{0}, z_{1}\right)\right] \\
& +k^{2}\left[\tau^{i+3} \rho\left(z_{0}, z_{1}\right)+\tau^{i+4} \rho\left(z_{0}, z_{1}\right)+\tau^{i+5} \rho\left(z_{0}, z_{1}\right)\right] \\
& +k^{3}\left[\tau^{i+6} \rho\left(z_{0}, z_{1}\right)+\tau^{i+7} \rho\left(z_{0}, z_{1}\right)+\tau^{i+8} \rho\left(z_{0}, z_{1}\right)\right]+\cdots \\
& +k^{j}\left[\tau^{i+2 j-3} \rho\left(z_{0}, z_{1}\right)+\tau^{i+2 j-2} \rho\left(z_{0}, z_{1}\right)+\tau^{i+2 j-1} \rho\left(z_{0}, z_{1}\right)\right]+k^{j} \tau^{i+2 j} \rho\left(z_{0}, z_{1}\right) \\
\leqslant & k \tau^{i}\left(e+k \tau^{3}+\left(k \tau^{3}\right)^{2}+\cdots\right) \rho\left(z_{0}, z_{1}\right)+k \tau^{i+1}\left(e+k \tau^{3}+\left(k \tau^{3}\right)^{2}+\cdots\right) \rho\left(z_{0}, z_{1}\right) \\
& +k \tau^{i+2}\left(e+k \tau^{3}+\left(k \tau^{3}\right)^{2}+\cdots\right) \rho\left(z_{0}, z_{1}\right) \\
= & k \tau^{i}\left(\sum_{j=0}^{\infty}\left(k \tau^{3}\right)^{j}\right)\left(e+\tau+\tau^{2}\right) \rho\left(z_{0}, z_{1}\right) \\
\therefore \quad \rho\left(z_{i}, z_{i+2 j+1}\right) \leqslant & k \tau^{i}\left(e-k \tau^{3}\right)^{-1}\left(e+\tau+\tau^{2}\right) \rho\left(z_{0}, z_{1}\right) .
\end{aligned}
$$

By using (7), we have $\left\|k \tau^{i}\left(e-k \tau^{3}\right)^{-1}\left(e+\tau+\tau^{2}\right) \rho\left(z_{0}, z_{1}\right)\right\| \rightarrow 0(i \rightarrow \infty)$, and it follows, by Lemma 2.3, that for any $c \in \mathcal{B}$ with $\theta \ll c$, there exists $n_{1} \in \mathbb{N}$ such that

$$
\rho\left(z_{i}, z_{i+2 j+1}\right) \preccurlyeq k \tau^{i}\left(e-k \tau^{3}\right)^{-1}\left(e+\tau+\tau^{2}\right) \rho\left(z_{0}, z_{1}\right) \ll c \text {, for all } i>n_{1} .
$$

Therefore, by Lemma 2.6(1), we get

$$
\rho\left(z_{i}, z_{i+2 j+1}\right) \ll c, \text { for all } i>n_{1},
$$

Case 1.2. If $n$ is even, say $n=2 j(j \in \mathbb{N})$, then, by using $\left(P b_{3}\right),(4),(5),(6)$ and the fact that $\rho\left(z_{i}, z_{i+1}\right) \neq \rho\left(z_{j}, z_{j+1}\right)$ whenever $i \neq j$, we obtain that

$$
\begin{aligned}
\rho\left(z_{i}, z_{i+2 j}\right) \leqslant & k\left[\rho\left(z_{i}, z_{i+1}\right)+\rho\left(z_{i+1}, z_{i+2}\right)+\rho\left(z_{i+2}, z_{i+3}\right)\right]+k \rho\left(z_{i+3}, z_{i+2 j}\right) \\
\leqslant & k\left[\rho\left(z_{i}, z_{i+1}\right)+\rho\left(z_{i+1}, z_{i+2}\right)+\rho\left(z_{i+2}, z_{i+3}\right)\right] \\
& +k^{2}\left[\rho\left(z_{i+3}, z_{i+4}\right)+\rho\left(z_{i+4}, z_{i+5}\right)+\rho\left(z_{i+5}, z_{i+6}\right)\right]+k^{2} \rho\left(z_{i+6}, z_{i+2 j}\right) \\
\leqslant & k\left[\rho\left(z_{i}, z_{i+1}\right)+\rho\left(z_{i+1}, z_{i+2}\right)+\rho\left(z_{i+2}, z_{i+3}\right)\right] \\
& +k^{2}\left[\rho\left(z_{i+3}, z_{i+4}\right)+\rho\left(z_{i+4}, z_{i+5}\right)+\rho\left(z_{i+5}, z_{i+6}\right)\right] \\
& +k^{3}\left[\rho\left(z_{i+6}, z_{i+7}\right)+\rho\left(z_{i+7}, z_{i+8}\right)+\rho\left(z_{i+8}, z_{i+9}\right)\right]+\cdots \\
& +k^{\frac{2 j-2}{3}}\left[\rho\left(z_{i+2 j-5}, z_{i+2 j-4}\right)+\rho\left(z_{i+2 j-4}, z_{i+2 j-3}\right)+\rho\left(z_{i+2 j-3}, z_{i+2 j-2}\right)\right] \\
& +k^{\frac{2 j-2}{3}} \rho\left(z_{i+2 j-2}, z_{i+2 j}\right)
\end{aligned}
$$




$$
\begin{aligned}
\rho\left(z_{i}, z_{i+2 j}\right) \leqslant & k\left[\tau^{i} \rho\left(z_{0}, z_{1}\right)+\tau^{i+1} \rho\left(z_{0}, z_{1}\right)+\tau^{i+2} \rho\left(z_{0}, z_{1}\right)\right] \\
& +k^{2}\left[\tau^{i+3} \rho\left(z_{0}, z_{1}\right)+\tau^{i+4} \rho\left(z_{0}, z_{1}\right)+\tau^{i+5} \rho\left(z_{0}, z_{1}\right)\right] \\
& +k^{3}\left[\tau^{i+6} \rho\left(z_{0}, z_{1}\right)+\tau^{i+7} \rho\left(z_{0}, z_{1}\right)+\tau^{i+8} \rho\left(z_{0}, z_{1}\right)\right]+\cdots \\
& +k^{j-1}\left[\tau^{i+2 j-5} \rho\left(z_{0}, z_{1}\right)+\tau^{i+2 j-4} \rho\left(z_{0}, z_{1}\right)+\tau^{i+2 j-3} \rho\left(z_{0}, z_{1}\right)\right]+k^{j-1} \tau^{i+2 j-2} \rho\left(z_{0}, z_{2}\right) \\
\leqslant & k \tau^{i}\left(e+k \tau^{3}+\left(k \tau^{3}\right)^{2}+\cdots\right) \rho\left(z_{0}, z_{1}\right)+k \tau^{i+1}\left(e+k \tau^{3}+\left(k \tau^{3}\right)^{2}+\cdots\right) \rho\left(z_{0}, z_{1}\right) \\
& +k \tau^{i+2}\left(e+k \tau^{3}+\left(k \tau^{3}\right)^{2}+\cdots\right) \rho\left(z_{0}, z_{1}\right)+k^{j-1} \tau^{i+2 j-2} \rho\left(z_{0}, z_{2}\right) \\
= & k \tau^{i}\left(\sum_{j=0}^{\infty}\left(k \tau^{3}\right)^{j}\right)\left(e+\tau+\tau^{2}\right) \rho\left(z_{0}, z_{1}\right)+k^{j-1} \tau^{i+2 j-2} \rho\left(z_{0}, z_{2}\right) \\
\leqslant & k \tau^{i}\left(e-k \tau^{3}\right)^{-1}\left(e+\tau+\tau^{2}\right) \rho\left(z_{0}, z_{1}\right)+k^{2 j-1} \tau^{2 j-2} \tau^{i} \rho\left(z_{0}, z_{2}\right) \\
\leqslant & k \tau^{i}\left(e-k \tau^{3}\right)^{-1}\left(e+\tau+\tau^{2}\right) \rho\left(z_{0}, z_{1}\right)+\tau^{i} \sum_{m=0}^{\infty} k^{m+1} \tau^{m} \rho\left(z_{0}, z_{2}\right) \\
\therefore \quad \rho\left(z_{i}, z_{i+2 j}\right) \leqslant & k \tau^{i}\left[\left(e-k \tau^{3}\right)^{-1}\left(e+\tau+\tau^{2}\right) \rho\left(z_{0}, z_{1}\right)+\left(\frac{e}{k}-\tau\right)^{-1} \rho\left(z_{0}, z_{2}\right)\right]
\end{aligned}
$$

By using (7), we have $\left\|k \tau^{i}\left[\left(e-k \tau^{3}\right)^{-1}\left(e+\tau+\tau^{2}\right) \rho\left(z_{0}, z_{1}\right)+\left(\frac{e}{k}-\tau\right)^{-1} \rho\left(z_{0}, z_{2}\right)\right]\right\| \rightarrow 0(i \rightarrow$ $\infty$ ), and it follows, by Lemma 2.3, that for any $c \in \mathcal{B}$ with $\theta \ll c$, there exists $n_{2} \in \mathbb{N}$ such that, for all $i>n_{2}$

$$
\rho\left(z_{i}, z_{i+2 j}\right) \preccurlyeq k \tau^{i}\left[\left(e-k \tau^{3}\right)^{-1}\left(e+\tau+\tau^{2}\right) \rho\left(z_{0}, z_{1}\right)+\left(\frac{e}{k}-\tau\right)^{-1} \rho\left(z_{0}, z_{2}\right)\right] \ll c .
$$

Therefore, by Lemma 2.6(1), we get

$$
\rho\left(z_{i}, z_{i+2 j}\right) \ll c, \text { for all } i>n_{2},
$$

Let $n_{0}:=\max \left\{n_{1}, n_{2}\right\}$, hence, from (8) and (9), we have that

$$
\rho\left(z_{i}, z_{i+n}\right) \ll c, \text { for all } i>n_{0},
$$

which implies, by Definition 3.4, that $\left\{z_{i}\right\}$ is a Cauchy sequence in $(\mathcal{Z}, \rho)$ and since $(\mathcal{Z}, \rho)$ is complete, the sequence $\left\{z_{i}\right\}$ converges i.e. there exists $z^{*}$ in $\mathcal{Z}$ such that $z_{i} \rightarrow z^{*}(i \rightarrow \infty)$. Next, we shall show that $z^{*}$ is a fixed point of $F$. By using $\left(P b_{3}\right),(1),(2)$ and (4), we have

$$
\begin{aligned}
\rho\left(z^{*}, F z^{*}\right) & \leqslant k\left[\rho\left(z^{*}, z_{i}\right)+\rho\left(z_{i}, z_{i+1}\right)+\rho\left(z_{i+1}, z_{i+2}\right)+\rho\left(z_{i+2}, F z^{*}\right)\right] \\
& \leqslant k\left[\rho\left(z^{*}, z_{i}\right)+\tau^{i} \rho\left(z_{0}, z_{1}\right)+\tau^{i+1} \rho\left(z_{0}, z_{1}\right)+\tau \rho\left(z_{i+1}, z^{*}\right)\right] \\
\therefore \rho\left(z^{*}, F z^{*}\right) & \leqslant k\left[\rho\left(z^{*}, z_{i}\right)+\tau^{i}(e+\tau) \rho\left(z_{0}, z_{1}\right)+\tau \rho\left(z_{i+1}, z^{*}\right)\right] \rightarrow \theta(i \rightarrow \infty) .
\end{aligned}
$$

Thus, $F z^{*}=z^{*}$. Hence, $z^{*}$ is a fixed point of $F$. Next we shall establish that this fixed point is unique. Assume that $y^{*}$ is another fixed point of $F$, i.e. $F y^{*}=y^{*}$, then using (1), we have that

$$
\rho\left(z^{*}, y^{*}\right)=\rho\left(F z^{*}, F y^{*}\right) \leqslant \tau \rho\left(z^{*}, y^{*}\right) .
$$

By lemma 2.6(2), we have that $\rho\left(z^{*}, y^{*}\right)=\theta$ and so $z^{*}=y^{*}$ i.e. the fixed point of $F$ is unique. Case 2. If $\delta(\tau) \in\left[\frac{1}{k}, 1\right)$ for $k>1$. Since $\delta(\tau)<1$ in this case, we have $[\delta(\tau)]^{i} \rightarrow \theta(i \rightarrow \infty)$, and so there exists $i_{0}$ such that $[\delta(\tau)]^{i_{0}}<\frac{1}{k}$. Note that $\delta\left(\tau^{i_{0}}\right) \leq[\delta(\tau)]^{i_{0}}<\frac{1}{k}$. Also by (1)

$$
\begin{aligned}
\rho\left(F^{i_{0}} z, F^{i_{0}} y\right) & =\rho\left(F\left(F^{i_{0}-1}\right) z, F\left(F^{i_{0}-1}\right) y\right) \leqslant \tau \rho\left(F^{i_{0}-1} z, F^{i_{0}-1} y\right)=\tau \rho\left(F\left(F^{i_{0}-2}\right) z, F\left(F^{i_{0}-2}\right) y\right) \\
& \leqslant \tau^{2} \rho\left(F^{i_{0}-2} z, F^{i_{0}-2} y\right) \leqslant \cdots \leqslant \tau^{i_{0}} \rho(z, y), \text { for all } i_{0} \in \mathbb{N} .
\end{aligned}
$$


Hence, by Case 1, the mapping $F^{i_{0}}$ has a unique fixed point $z^{* *}$ in $\mathcal{Z}$. Now, observe that

$$
F^{i_{0}}\left(F z^{* *}\right)=F^{i_{0}+1}\left(z^{* *}\right)=F\left(F^{i_{0}} z^{* *}\right)=F z^{* *},
$$

that is, $F z^{* *}$ is also a fixed point of $F^{i_{0}}$. Thus, by uniqueness of the fixed point of $F^{i_{0}}$, we get that $F z^{* *}=z^{* *}$, which imply that $z^{* *}$ is also a fixed point of $F$. Now suppose that $z^{* * *} \in \mathcal{Z}$ is another fixed point of $F$ i.e. $F z^{* * *}=z^{* * *}$. Then

$$
\begin{aligned}
F^{i_{0}} z^{* *}=F^{i_{0}-1}\left(F z^{* *}\right) & =F^{i_{0}-1} z^{* *}=\cdots=F z^{* *}=z^{* *}, \\
F^{i_{0}} z^{* * *}=F^{i_{0}-1}\left(F z^{* * *}\right) & =F^{i_{0}-1} z^{* * *}=\cdots=F z^{* * *}=z^{* * *},
\end{aligned}
$$

which means that $z^{* *}$ and $z^{* * *}$ are fixed point of $F^{i_{0}}$. Thus, by uniqueness of the fixed point of $F^{i_{0}}$, we get that $z^{* *}=z^{* * *}$ i.e. the fixed point of $F$ is only one of its kind.

Case 3. If $k=1$. Since $\delta(\tau)<1$, the proof follows from Case 1. This completes the proof.

Example 3.6 In Example 3.3, $(\mathcal{Z}, \rho)$ is a pentagonal cone b-metric space over Banach algebra $\mathcal{B}$ with coefficient $k=\left(\begin{array}{ll}2 & 0 \\ 0 & 2\end{array}\right)$. Now we define a mapping $F: \mathcal{Z} \rightarrow \mathcal{Z}$ as follows:

$$
F z= \begin{cases}4, & \text { if } z \neq 5 \\ 2, & \text { if } z=5\end{cases}
$$

Hence, $\rho(F 1, F 2)=\rho(F 1, F 3)=\rho(F 1, F 4)=\rho(F 2, F 3)=\rho(F 2, F 4)=(F 3, F 4)=$ $\left(\begin{array}{ll}0 & 0 \\ 0 & 0\end{array}\right)$. And in all other cases we have that $\rho(F z, F y)=\left(\begin{array}{cc}\frac{1}{2} & \frac{1}{2} \\ 1 & 1\end{array}\right), \rho(z, y)=\left(\begin{array}{ll}2 & 2 \\ 3 & 3\end{array}\right)$. Let $\tau \in \mathcal{P}$ where $\tau:=\left(\begin{array}{cc}\frac{1}{3} & 0 \\ 0 & \frac{1}{3}\end{array}\right)$. It is easy to prove that $\|\tau\|<1$, which implies that $\delta(\tau)<1$. Hence, the mapping $F$ satisfies all the conditions of Theorem 3.5 and $z^{*}=4 \in \mathcal{Z}$ is the only one of its kind fixed point of $F$.

\section{Conclusion}

In this piece of writing, we instigate the idea of pentagonal cone $b$-metric space over Banach algebras and prove Banach fixed point theorem in such a space. Our result, Theorem 3.5, generalizes Theorem 2.1 in [1], Theorem 3.1 in [2], Theorem 2.1(i) in [3], Theorem 5 in [4], Theorem 3.5 in [5], Theorem 3.10 in [6], and Theorem 3.1 in [7] to a more general pentagonal cone $b$-metric space over a Banach algebra. We hope that this result will be useful in fixed point theory and may be generalized in further spaces with efficient conditions.

\section{References}

[1] L. Huang, Xu. Shao, Fixed Point Theory Appl. 320, 1-10 (2013)

[2] S. Xu, S. Radenović, Fixed Point Theory Appl. 102, 1-12 (2014)

[3] H. Huang, S. Radenović, J. Comput. Anal. Appl. 20, 566-583 (2016)

[4] S. Shukla, S. Balasubra, M. Pavlovic, Bull. Mal. Math. Sci. Soc. 39, 1529-1539 (2016)

[5] R. George, H.A. Nabwey, R. Raj, S. Radenović, Fixed Point Appl. 14, 1-15 (2017)

[6] M. Garg, S. Agarwal, J. Adv. Stud. Topol. 3, 12-18 (2012)

[7] A. Auwalu, E. Hınçal, Int. Conf. Anal. Appl. Math. (ICAAM), (Accepted) (2018)

[8] W. Rudin, Functional Analysis (McGraw-Hill Inc., New York, 1991) 245-272 